\title{
Dynamics of a Four-Bar mechanism with Clearance and Springs - Modeling and Experimental Analysis
}

\author{
M. Ahmedalbashir, L. Romdhane*, J. Lee \\ Mechanical Engineering Department, College of Engineering, American University of Sharjah, UAE \\ Journal of Mechanical Science and Technology 31 (3) (2017) 1 11 \\ www.springerlink.com/content/1738-494x(Print)/1976-3824(Online) \\ DOI 10.1007/s12206-016-0913-y
}

\begin{abstract}
This paper presents a study of the dynamic behavior of a planar four-bar mechanism with clearance. A spring is added to improve the dynamic response of the mechanism with clearance. Simulations and experimental tests are carried out to show the improvement of the dynamic behavior using this simple yet effective solution. The simulation is performed under MATLAB and ADAMS. An experimental set-up is designed and built to achieve the experimental validations. The presented results show that the added spring improved the dynamic behavior of the mechanism by keeping a continuous contact between the journal and the sleeve despite the presence of the clearance. The effect of the spring on the input torque is also investigated. It is shown that the spring increases the maximum value of the torque. An optimization is performed to determine the best parameters of the spring that reduce the effect of the clearance while minimizing the increase of the input torque. The optimized system is tested at different speeds to show the effect of the speed on the dynamic behavior of the optimized system.

Key Words: Four-Bar Mechanism; Clearance in the Joint; Experimental Testing; MATLAB Simulation; ADAMS; Spring; Optimization
\end{abstract}

\section{Introduction}

The dynamic analysis of mechanisms with clearance has been frequently studied in the literature for the last three decades. Indeed, several studies showed that the presence of clearance in the joints degrades the performances of the mechanism and generally leads to the presence of raised constraints, vibration and noise ([1], [2]).

The literature in this domain contains several works dealing with the development of suitable formulations of the dynamic model of mechanisms such as the four-bar system and the slider-crank mechanism. These studies presented analytical and experimental results to show the effect of the clearance on the dynamic behavior of these mechanisms ([1] - [14]).

To model and predict the effect of the clearance on the dynamic behavior of planar mechanisms, Flores et al. proposed approaches based on the physics of the contact between the two bodies forming the joint [1]. The method presented in [1] is based on geometrical constraints. A continuous contact force model, based on the elastic Hertz theory, together with a dissipative term associated with the internal damping, is utilized to evaluate the joint normal contact forces. The authors included also the friction, based on Coulomb's friction law.

The authors of [7], [15], and [16] presented a computational methodology for dynamic analysis of multibody mechanical systems with joint clearance based on a continuous contact force model. They presented a strategy to detect the contact that overcomes the problems raised by the discontinuity of the problem during impact. An extensive combined experimental analytical study was conducted in [13] to validate the proposed joint clearance models.

The software ADAMS was also extensively used to simulate mechanisms with clearance. ADAMS uses a contact model based on the so called Impact-function ([3], [4], [16]). This model is similar to the continuous contact force model.

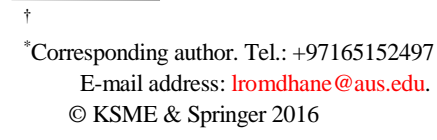

Real mechanisms having clearance in the joints can also have flexible links [3], [4], [10], [11], [14], [16], [17], [18].

The combination of these two imperfections can have a great negative effect on the dynamic behavior of real mechanisms. It was shown in [3], for example, that in the presence of clearance, the coupler flexibility has a role of suspension for the mechanism.

Dynamic models of mechanisms with clearance are usually complex and involve many assumptions. In order to validate these models experimental results are required. Several studies, found in the literature, conducted experiments to validate their models ([3], [4], [10], [11], [12], [13], [14], [16], [17]). Hai-yang et al. [17] used experimental results to verify the simulation results obtained using oversized joint clearance fault in one of the joints of the studied compressor. The experimental results were obtained through the measurement of the acceleration in different points of the system. These results were compared to the simulation results to validate the model.

To reduce the effect of the clearance, several authors formulated the problem as an optimization problem ([14], [16], [19], [20], [21], [22], [23], [24], [25], [26]). Singleobjective and multi-objective optimization problems were addressed. Feng et al. [24] and Varedi et al. [20] minimized the impact forces by redistributing the mass of the different links. In [19], Zhang et al. considered four objective functions to be optimized simultaneously, i.e., the slider's acceleration, the contact force at the revolute joint clearance, the required input torque, and the power consumption of the system. The design variables to be found are the size of the clearance and the stiffness of the contact. Erkaya et al. studied the effect of the clearance in the joints on the characteristics of a 4R mechanism [21], and a slider-crank mechanism [11]. A neural-genetic (NN-GA) approach for optimizing mechanisms having joints with clearance was proposed in [22]. The neural network (NN) method was used 
to define the characteristics of the joints with clearance with respect to the position of the input link, and the genetic algorithm (GA) was used to implement the optimization of link parameters using an appropriate objective function based on path and transmission angle errors. The trajectory of a walking mechanism having revolute joints with clearance was optimized in [23]. The adaptive networkbased fuzzy inference system (ANFIS) approach was used to model the characteristics of the joints with clearance [23]. Yaqubi et al. [6] proposed adding an extra actuator in order to avoid the impacts at the joint with clearance in order to improve its dynamic behavior. Hai-yang et al. [17] used ADAMS to calculate their objective function required by the genetic algorithm method. Their objective was to find the best parameters of the joint clearance in order to fit the simulated dynamic response to the experimental results.

In this work, we are interested in the dynamic response of a planar rigid four-bar mechanism with clearance. In order to improve the dynamic behavior of the mechanism, the reaction force in the joint with clearance is modified such that the contact is never lost, hence avoiding any impact within the joint. This modification is performed by attaching a spring to the mechanism, which has the advantage of being simple to implement and does not require any modification of the original mechanism. Several tests are performed to determine the validity of the proposed method, which lead to an optimization of the location of the spring.

The manuscript is organized as follows: in Section 2, we present the model for the mechanism with clearance and simulate its effect without and with a spring attached. In Section 3, we describe the experimental set-up designed and built to carry out some experimental validations. Section 4 deals with the optimization of the location of the spring in order to minimize the maximum torque while maintaining the contact inside the joint with clearance. Section 5 contains some concluding remarks.

\section{Modeling and Simulation of the System}

\subsection{Description of the mechanism}

Fig. 1 shows the four-bar mechanism with a clearance, $e$, in the revolute joint connecting the coupler to the follower.

The four-bar mechanism is made of a rigid crank, a rigid coupler, and a rocking follower. The crank rotates at a constant angular velocity of $277 \mathrm{rpm}$. The mechanism characteristics are presented in Table 1. The spring is attached to points $\mathrm{B}_{3}$ and $\mathrm{B}_{4}$.

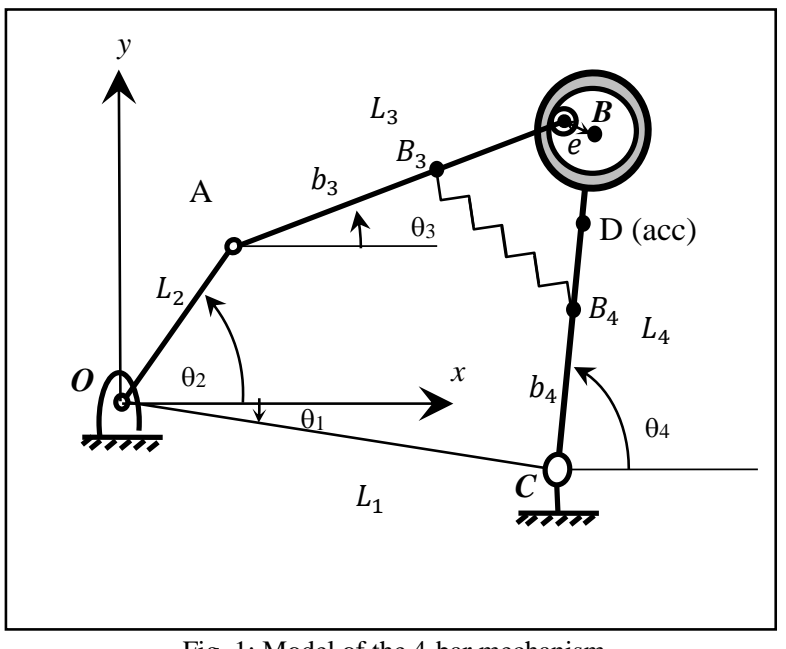

Fig. 1: Model of the 4-bar mechanism

Table 1: Mechanism parameters

\begin{tabular}{|c|c|c|c|}
\hline Links & $\begin{array}{l}\text { Geometry } \\
\quad(\mathbf{m m})\end{array}$ & inertia & $\begin{array}{l}\text { Center of } \\
\text { mass }\end{array}$ \\
\hline $\begin{array}{l}\text { The ground } \\
\text { (1) }\end{array}$ & $L_{1}=410$ & & \\
\hline $\begin{array}{l}\text { The crank } \\
\text { (2) }\end{array}$ & $L_{2}=100$ & $\begin{array}{l}m_{2}=1 \mathrm{Kg} \\
I_{2}=0.0023 \mathrm{Kgm}^{2}\end{array}$ & $r_{g 2} \approx 0$ \\
\hline $\begin{array}{c}\text { The } \\
\text { coupler (3) }\end{array}$ & $L_{3}=330$ & $\begin{array}{c}m_{3}=0.284 \mathrm{Kg} \\
I_{3}=0.035 \mathrm{Kgm}^{2}\end{array}$ & $r_{g 3}=L_{3} / 2$ \\
\hline $\begin{array}{c}\text { The } \\
\text { follower } \\
(4) \\
\end{array}$ & $L_{4}=230$ & $\begin{array}{c}m_{4}=0.106 \mathrm{Kg} \\
I_{4}=0.006 \mathrm{Kgm}^{2}\end{array}$ & $r_{g 4}=L_{4} / 2$ \\
\hline \multicolumn{4}{|c|}{ Accelerometer in point $\mathrm{D}, \mathrm{CD}=210 \mathrm{~mm}$} \\
\hline Spring & $\begin{array}{c}\text { Stiffness } k= \\
279 \mathrm{~N} / \mathrm{m} \\
\text { Unstretched } \\
\text { length } \\
s_{0}=110 \\
\mathrm{~mm}\end{array}$ & $\begin{array}{c}\mathrm{AB}_{3}=b_{3} \\
\text { (to be determined) }\end{array}$ & $\begin{array}{c}\mathrm{CB}_{4}=b_{4} \\
(\text { to be } \\
\text { determined) }\end{array}$ \\
\hline
\end{tabular}

\subsection{Modeling of the clearance}

In the revolute joint, connecting the coupler to the follower, the clearance is modeled by an introduction of two additional degrees of freedom, the horizontal and the vertical displacements, $x$ and $y$, of the journal center relative to the sleeve center (Fig. 2). The journal diameter is $8 \mathrm{~mm}$ while the sleeve diameter is $9 \mathrm{~mm}$.

During the contact, the interaction between the two parts in the joint is only carried out by the normal and the tangential forces of contact. The kinematic contact condition for a revolute joint with radial clearance $e$ and relative displacements, $x$ and $y$, is given by:

$$
g=e-\sqrt{x^{2}+y^{2}} \leq 0
$$

The situation where the contact is lost corresponds to $g>0$ (Fig. 2), whereas the contact with local deformation, referred to as penetration, corresponds to $g<0$.

The reaction force between link 3 (coupler) and link 4 (follower), $\boldsymbol{R}_{\mathbf{3 4}}$, if oriented in the right direction, maintains the contact between the journal and the sleeve. During the motion, however, this direction changes and could lead to a loss of the contact and probably to an impact of the journal against the sleeve on the side of the initial contact. Therefore, monitoring the reaction force could explain the occurrence of a possible impact. Fig. 3(a) shows the reaction force $\boldsymbol{R}_{\mathbf{3 4}}$ 
which maintains the contact in point $\mathrm{H}$. It is worth mentioning that the surface of contact is always normal to the reaction force. This side of contact is maintained as long as the direction of the force $\boldsymbol{R}_{\mathbf{3 4}}$ induces a positive moment around A. If, however, the direction changes due to the dynamic of the overall system, such that the moment around point A becomes negative, then the contact is lost and the journal gets ejected against the other side of the sleeve (Fig. 3 (b)). Therefore, the side of the contact journal-sleeve is dictated by the position of the reaction force $\boldsymbol{R}_{\mathbf{3 4}}$, with respect to the bar $\mathrm{AB}$.

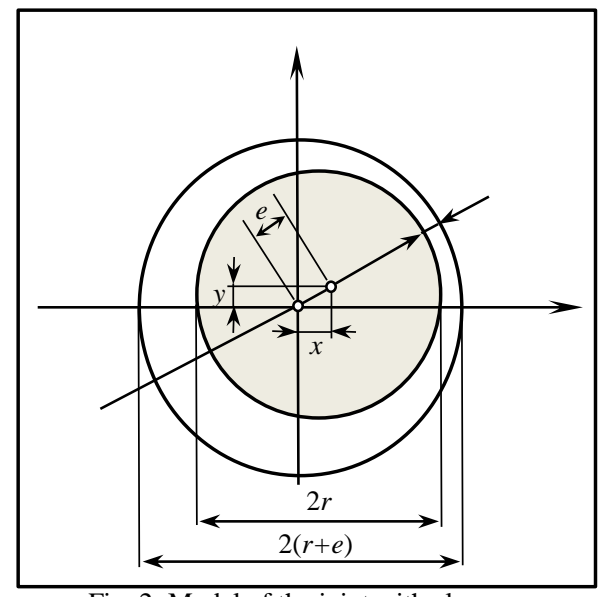

Fig. 2: Model of the joint with clearance

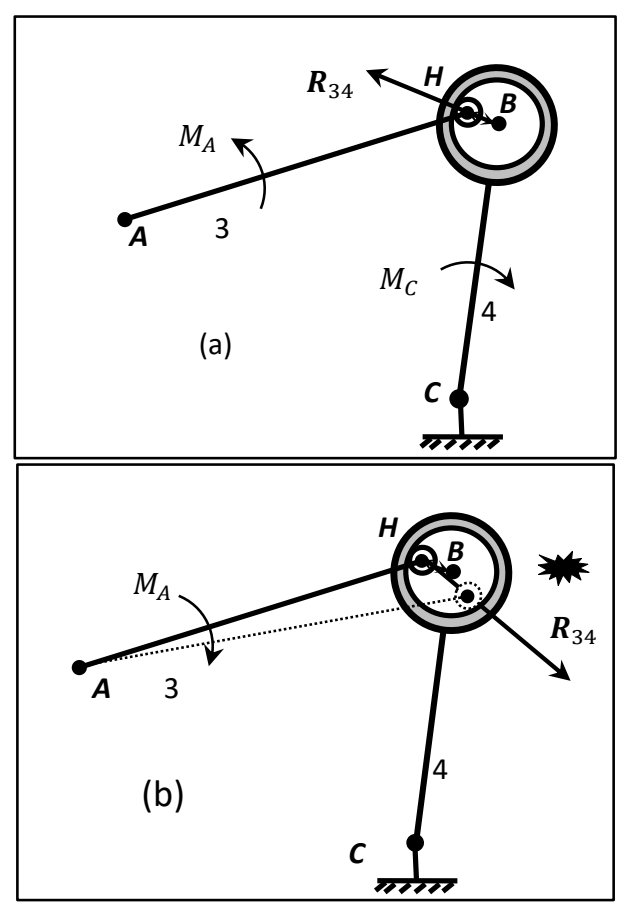

Fig. 3: The direction of the reaction force $\boldsymbol{R}_{34}$ (a) at an instant $t$ and (b) at $\mathrm{t}+\mathrm{dt}$

Since the clearance is small compared to the displacement of point $\mathrm{B}$, we will assume that the change of side is instantaneous. Therefore, to detect the impact, one has to monitor the moment of the reaction force $\boldsymbol{R}_{\mathbf{3 4}}$ around point A, $\boldsymbol{M}_{\boldsymbol{A}}$

$M_{A}=A B \times R_{34}$

$\boldsymbol{A B}$ and $\boldsymbol{R}_{34}$ are given in the Appendix.

In the example presented in Fig. 3(a), $M_{A}$ is positive. This situation ensures the contact between the sleeve (Link 3) and the journal (Link 4). In Fig. 3(b), $M_{A}$ becomes negative yielding a loss of the contact between the sleeve (Link 3) and the journal (Link 4). At this instant, the link 4 continues its motion under the inertia forces for a brief instant until the journal impacts the sleeve on the other side. This period of free flight is short and one can assume that the impact occurs as soon as the moment $M_{A}$ changes its sign.

Since the input of the mechanism is the torque applied to the crank (Link 2), which drives the coupler (Link 3) and determines its dynamics, one needs to monitor only the moment $M_{A}$. The change of the sign of this moment yields the impact in the joint with clearance.

Therefore, to eliminate any impact in the joint with clearance, one has to maintain the sign of the moment $M_{A}$ always the same. The proposed solution is to add a spring to the system to ensure that the contact is maintained at all times.

First, the kinetostatic model of the mechanism with ideal joints and without a spring is solved (see Appendix). Then the moment $\boldsymbol{M}_{\boldsymbol{A}}$ is plotted as a function of time. The introduction of a clearance in the joint in B, will not modify the overall dynamics of the system, however it will introduce some discontinuities (peaks) in the response due to the impact that could occur in the joint with clearance. Based on the value of the moment $\boldsymbol{M}_{\boldsymbol{A}}$, one can predict these impacts, by monitoring the sign of $\boldsymbol{M}_{\boldsymbol{A}}$. To avoid these impacts, we propose to add a spring to the system, in order to keep the continuous contact in the joint with clearance. To minimize the side effect of adding a spring on the input torque, the optimum locations of the attachment points are determined.

\subsection{Simulation Results}

Fig. 1, shows the four-bar mechanism used in the simulations. First, the dynamic model of the system is derived (Appendix). All the links are assumed to be rigid and all the joints are without clearance. Following the experimentation, a sampling time of $t=0.244 \mathrm{~ms}$, is used in the simulation, which corresponds to a step of $d \theta_{2}=0.4^{\circ}$ in the crank rotation, for a speed of $\omega_{2}=29 \mathrm{rad} / \mathrm{s}$.

The angular velocity of the crank is assumed to be constant during the motion. Only inertia forces and the input torque are applied to the system.

Fig. 4 shows a simulation of the mechanism where a vector representing the reaction force, $\boldsymbol{R}_{\mathbf{3 4}}$, is shown. For more clarity the mechanism is drawn only in 12 different positions $(i=1,12)$. This simulation shows that the reaction force is variable in magnitude and direction during the motion (Fig. 4).

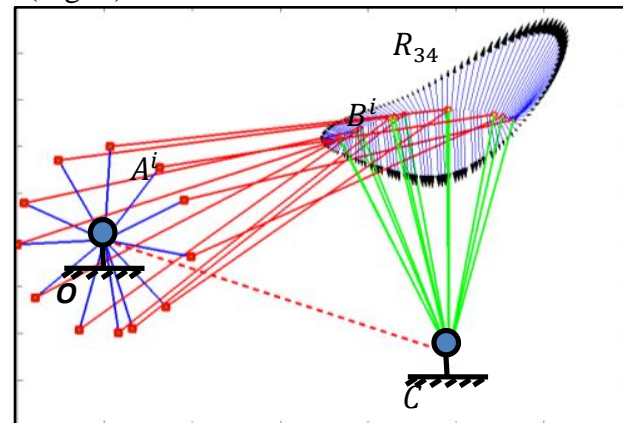

Fig. 4: Simulation of the four-bar mechanism showing the variation of the vector $R_{34}$ during the motion of the crank.

Fig. 5 shows the variation of the moment of the reaction force in the joint around point $\mathrm{A}, \boldsymbol{M}_{\boldsymbol{A}}=\boldsymbol{A B} \times \boldsymbol{R}_{34}$. It is 
worth mentioning that the value of the moment $M_{A}$ changes sign twice during one rotation of the crank.

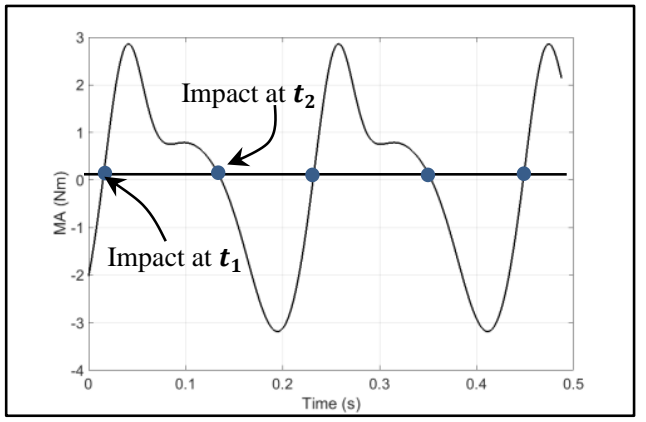

Fig. 5: Moment of the reaction force around point $\mathrm{A}$

Fig. 6 shows the mechanism for two values of the input angle. The first one $A^{1}$ corresponds to the instant just before the impact, the second one just after the impact. The reaction force $\boldsymbol{R}_{\mathbf{3 4}}$ is also plotted for these two configurations. The relative position of the reaction force $\boldsymbol{R}_{\mathbf{3 4}}$ with respect to link 3 (AB) determines the sign of the moment $\boldsymbol{M}_{\boldsymbol{A}}$. At the instant where the moment $\boldsymbol{M}_{\boldsymbol{A}}$ becomes zero, the contact between the journal and the sleeve in joint B is lost and the sleeve is ejected against the other side of the sleeve due to the change of sign of the moment $\boldsymbol{M}_{\boldsymbol{A}}$ on link 3. This change generates a brief impact between the sleeve and the journal, causing the acceleration of any point on link 3 and link 4 to surge to a high value.

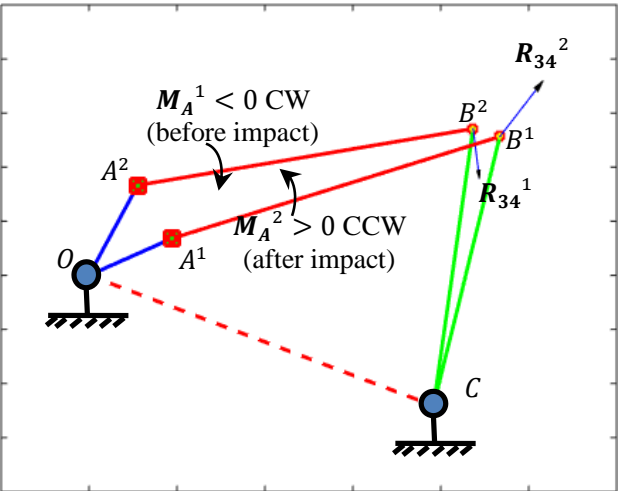

Fig. 6: Configurations of the mechanism around the instant of the change of the sign of $M_{A}$ from a positive value to a negative value (instant $t_{1}$ from Fig. 5)

Fig. 7 shows the second occurrence of the change of sign during a full rotation of the crank. The same remarks, made for Fig. 6, are valid in this case. The difference, however, is in the change of direction of the moment $\boldsymbol{M}_{\boldsymbol{A}}$. In this case, the moment $\boldsymbol{M}_{\boldsymbol{A}}$ changes direction from $\mathrm{CCW}$, before impact, to $\mathrm{CW}$, after impact.

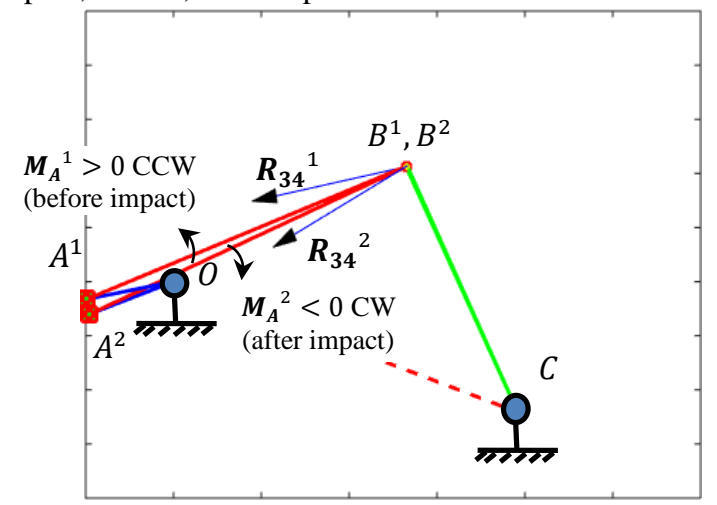

Fig. 7: Configurations of the mechanism around the instant of the change of the sign of $M_{A}$ from a negative value to a positive value (instant $t_{2}$ from Fig. 5)

\section{Experimental validation}

\subsection{Four-bar mechanism with clearance}

To validate the simulation results based on the model presented previously, an experimental setup was designed and built. It consists of a four bar mechanism driven by a DC motor. The geometric and inertia parameters of the mechanism are listed in Table 1. The experimental setup is shown on Fig. 8.

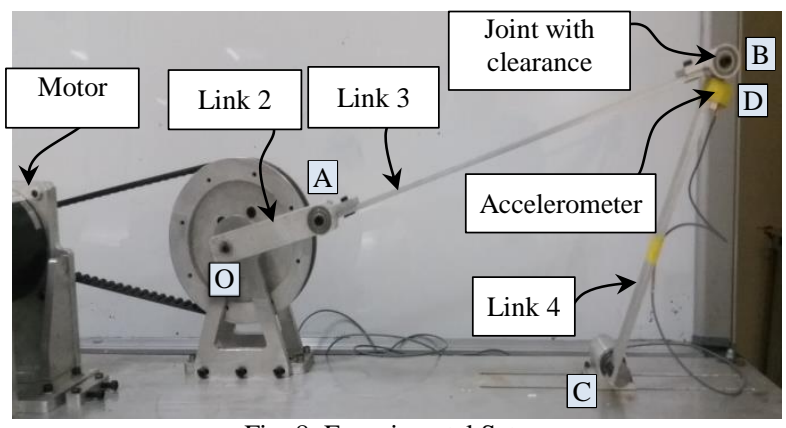

Fig. 8: Experimental Setup

The revolute joint connecting the coupler to the follower is built so a clearance of $1 \mathrm{~mm}$ could be introduced. This exaggerated clearance of $1 \mathrm{~mm}$ is chosen to accentuate the effect of the impact and allow an easy measurement of the effect of the impact.

A data acquisition system made out of a tachometer, an accelerometer, and a current sensor, was used. The tachometer is used to measure the speed of the motor. The rotation of the crank is ensured, through a pulley pair having a transmission ratio of 3 , by a variable speed D.C permanent magnet servo motor.

A flywheel, having a moment of inertia of $0.026 \mathrm{~kg} / \mathrm{m}^{2}$, is mounted in order to reduce the speed fluctuation of the input link. The accelerometer is used to measure the acceleration near the joint with clearance (point D). All these measurements are made simultaneously during one second of motion, which yields 4096 samples at a sampling time of $t_{s}=0.244 \mathrm{~ms}$.

The experimental validation is made through the measurement of the acceleration near the joint with clearance. Fig. 9 and Fig. 10 show a comparison between the measured acceleration and the simulated one in point $\mathrm{D}$, when the joint between link 3 and 4 is without clearance and with clearance (Fig. 8). The crank speed is $277 \mathrm{rpm}$.

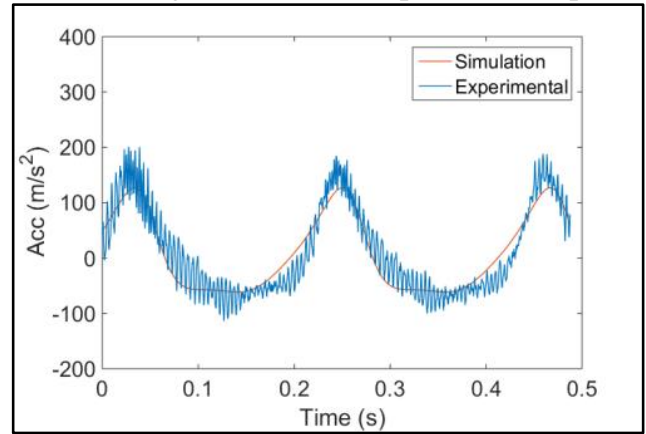

Fig. 9: Experimental and analytical results of the acceleration in the case with no clearance 


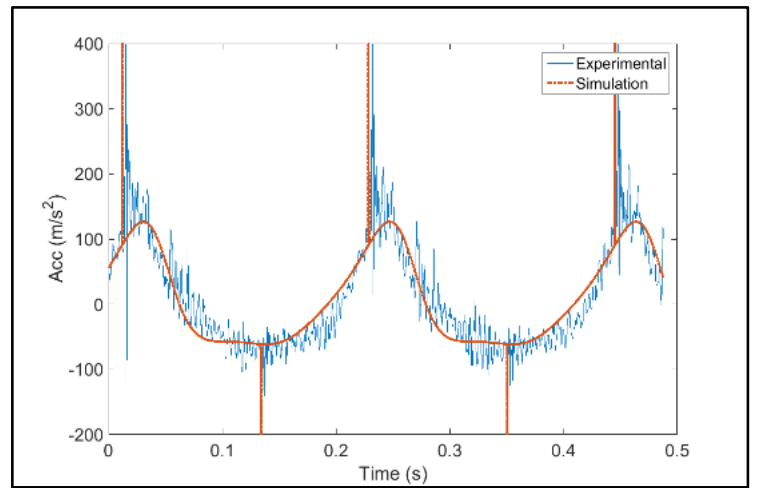

Fig. 10: Experimental and simulation results with clearance in the joint

In Fig. 10, the simulated acceleration (in red) is modified artificially by adding a peak every time the moment $\boldsymbol{M}_{\boldsymbol{A}}$ changes sign. One can notice that the occurrence of the impact (experimental) coincides with the change in the sign of the moment $\boldsymbol{M}_{\boldsymbol{A}}$ (simulation). It is worth mentioning that the magnitude of the introduced peak is arbitrary and the model presented in the Appendix is not capable of calculating it. Indeed, the kinetostatic model is based on an ideal mechanism with no clearance. However, the time of the occurrence of the impact could be predicted, using this simple kinetostatic model, if one monitors the sign of the moment $\boldsymbol{M}_{\boldsymbol{A}}$. This result is in line with the proposed result in the literature [12]. Since the objective here is to identify the cause of the impact, the results presented in Fig. 5 and Fig. 10 show clearly that there is a close correlation between the change of the direction of the moment of the reaction force around point A (Fig. 1) and the occurrence of the impact.

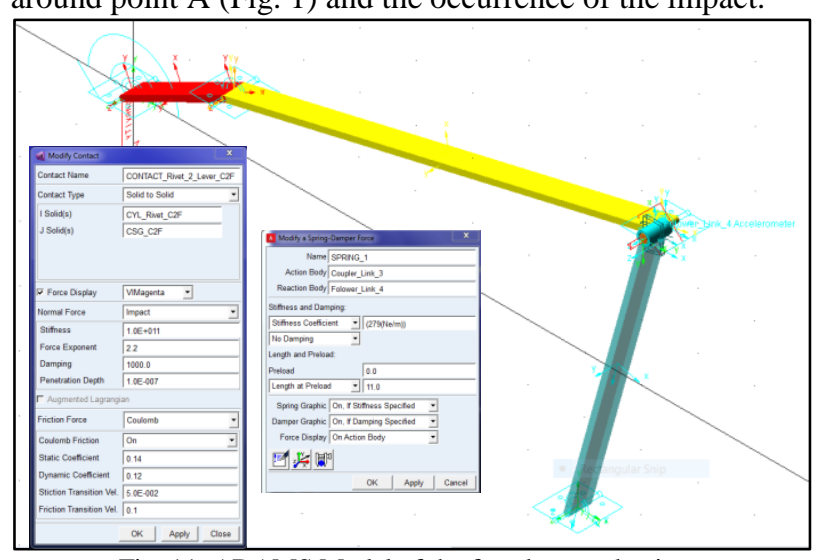

Fig. 11: ADAMS Model of the four-bar mechanism

To be able to model the joint with clearance, the software ADAMS was used. This model along with the parameters used for the simulation of the joint with clearance, are shown Fig. 11. The model of the joint with clearance can be deactivated if one needs to simulate the mechanism without clearance.

This model requires also the input of the type and the parameters of the friction model. In the literature ([1], [2], [3], [20]) most authors recommend the Coulomb model in the case of a revolute joint. The obtained results are sensitive to this friction model.

Fig. 12 shows the obtained acceleration of point $D$, using ADAMS model. One can notice the presence of high frequency components, which could be attributed to the friction between the journal and the sleeve.

Fig. 13 shows the trajectory of the center of the journal, with respect to the bearing, corresponding to one rotation of the crank. This plot is obtained using an ADAMS model
(Fig. 11), where only a few cycles from the steady state motion are shown. The trajectory is obtained by monitoring the difference between the position of the center of the journal and the center of the bearing, during the motion of the mechanism. As seen from the figure, the different types of motion between the journal and the bearing are shown, i.e., the continuous contact, free-flight and impact. Similar results were obtained by several authors [1], [2], [3], [4], for example.

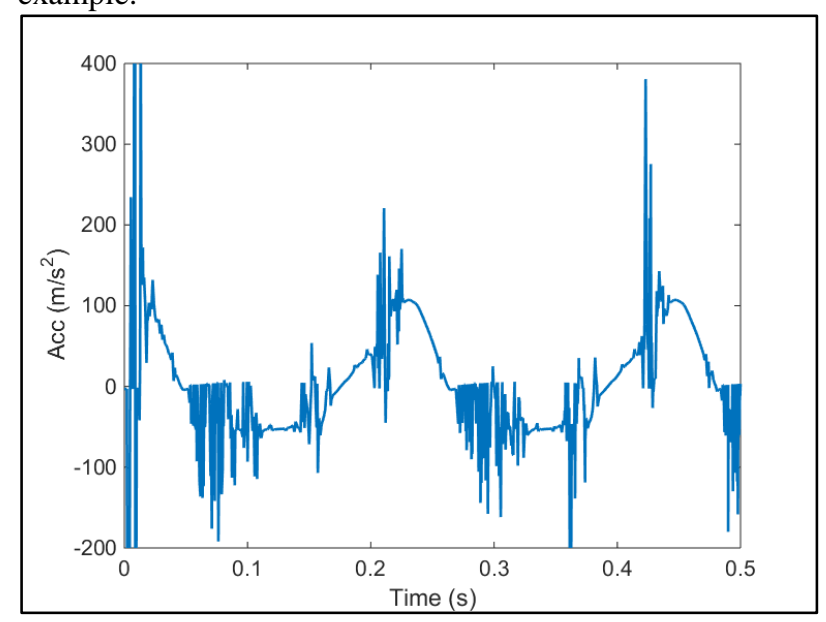

Fig. 12: Acceleration given by ADAMS simulation

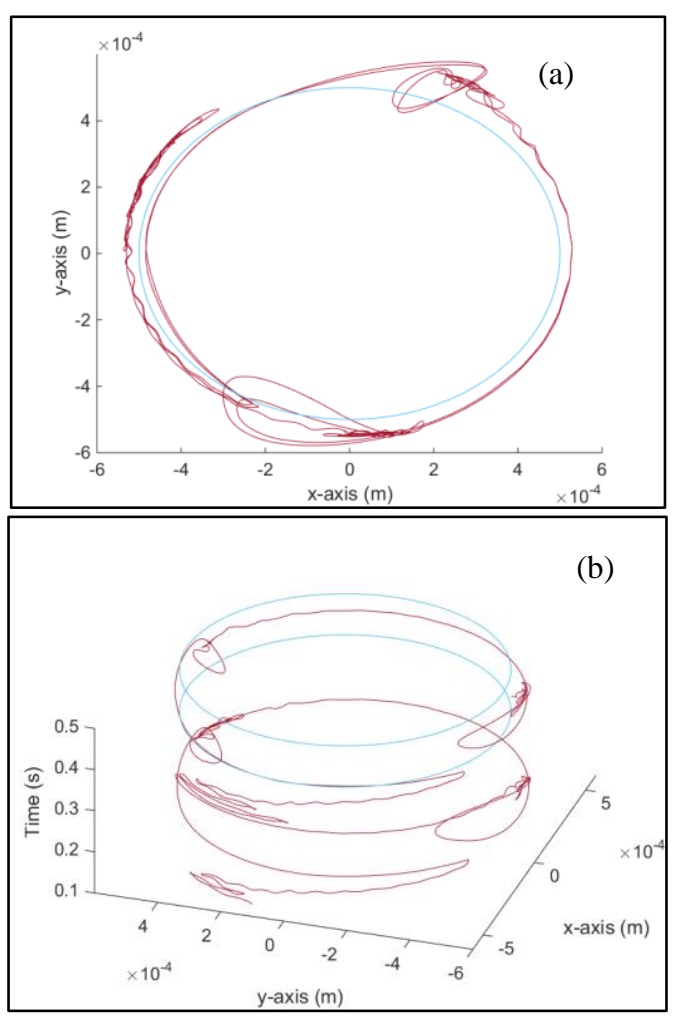

Fig. 13: ADAMS model: journal center trajectory (a) XY plot, (b) XYtime plot (the circles represent the clearance)

\subsection{Four-bar mechanism with a spring}

To reduce the effect of the clearance on the dynamic behavior of the mechanism, several authors used optimization techniques to find the best parameters of the mechanism. The authors of [19] used the clearance in the joint and the stiffness of the material as design variables to improve the dynamic response of a crank slider mechanism. The authors of [21], [22], [23], and [25] proposed modifying 
the geometry of the mechanism to find the best geometric parameters yielding an optimized mechanism with clearance. The authors of [20] and [24] proposed to modify the mass distribution of the different elements to reduce the change of forces due to clearance. Yaqubi et al. [6] proposed adding a second actuator to keep a permanent contact in the joint with clearance. This solution, even though was shown to be effective, is too complex and expensive to implement. All these studies required the modification of the parameters of the mechanism. In this work a simple solution, based on the use of springs, is proposed, which requires a minimal modification of the existing system. Since the loss of the contact in the joint with clearance is caused by the reaction force $\boldsymbol{R}_{\mathbf{3 4}}$ changing sides relative to the coupler link (AB), an added spring can avoid this change and hence maintain a permanent contact in the joint with clearance. However, adding a spring requires an extra amount of torque to drive the mechanism. Therefore, an optimization of the locations of the attachment points of the spring have to be performed, in order to minimize the value of the added torque while maximizing the reduction of the impact. In this section, an analysis of the effect of adding a spring to the system is analyzed.

The mechanism has now a spring attached between the coupler and the follower (Fig. 1). The objective is to find the best location of a spring having a stiffness of $k=279 \mathrm{~N} / \mathrm{m}$ capable of keeping the moment $\boldsymbol{M}_{\boldsymbol{A}}$ from changing sign. Fig. 14 shows the value of the reaction of the moment of $\boldsymbol{R}_{\mathbf{3 4}}$ around point $\mathrm{A}, \boldsymbol{M}_{\boldsymbol{A}}$. Introducing the spring resulted in an increase of the absolute value of the minimum and the mean of this moment. This increase induces more stress in the joint, which can be considered as a negative effect. In the case of the spring attached in the middle of the two links (curve 2), the moment still has positive and negative values, which means that the reaction force changes direction in the joint with clearance. This change causes impacts in the joint. When the spring is attached in another location (curve 3), the sign of the moment $\boldsymbol{M}_{\boldsymbol{A}}$ is maintained constant (negative) during the motion. In this location the spring prevents the impact from occurring in the joint with clearance.

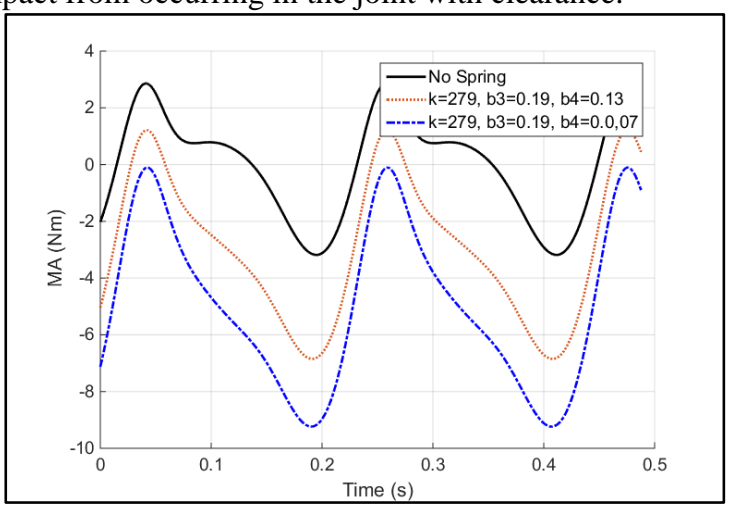

Fig. 14: Moment around point A of the reaction force, with and without a spring

The second effect of the spring is on the input torque required to drive the system. Fig. 15 shows the values of the input torque in the three previous cases. When the spring is attached in the middle of the two links (curve 2), the simulations show that the input torque increases compared to the case without a spring. However, as can be seen from Fig. 14, the moment of the reaction force, $\boldsymbol{M}_{\boldsymbol{A}}$ still changes sign. If the same spring is attached in different locations on the two links (curve 3 ), the values of the input torque can reach high values but the moment $\boldsymbol{M}_{\boldsymbol{A}}$ keeps the same sign, in this case (Fig. 14, Fig. 15).

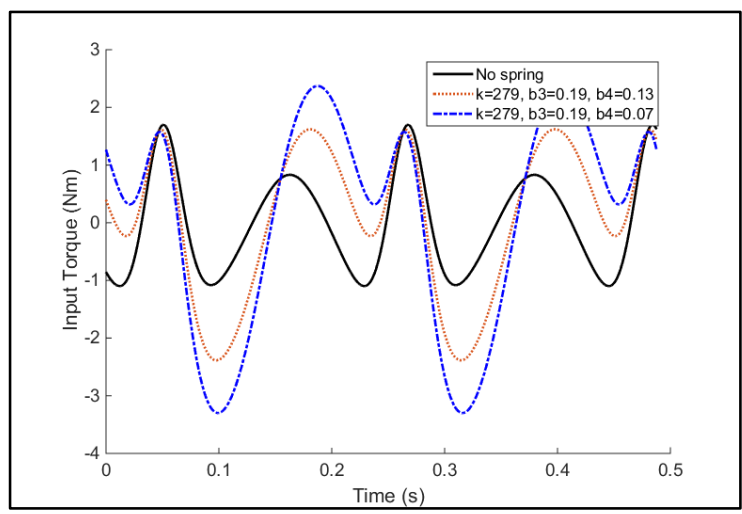

Fig. 15: Input torque with and without a spring

Therefore, the spring has positive and negative effects on the dynamic behavior of the system. The objective of the next section is to optimize the parameters of the spring to be added, in order to maximize its positive effect while minimizing the negative one.

\section{Optimization of the parameters of the spring}

In the following, an optimization procedure is performed, in order to find the best values of the parameters of the spring. These parameters are defined as attachment points of the spring, $b_{3}$ and $b_{4}$ (Fig. 1). The stiffness of the spring is taken as $k=279 \mathrm{~N} / \mathrm{m}$ and its pre-tension, represented by the unstretched length, is $s_{0}=110 \mathrm{~mm}$.

\subsection{Formulation of the problem}

The objective function to be minimized is the peak value of the torque. MATLAB function "fmincon" was used to solve the optimization problem for minimizing the maximum torque. The optimization problem can be formulated as follows:

Objective function:

$$
F=\min \left(T_{\max }\right)(\text { over a full cycle) }
$$

where $T_{\max }$ is the maximum torque calculated in the Appendix.

Subject to:

$$
\begin{gathered}
0<b_{3}<L_{3} \\
0<b_{4}<L_{4} \\
M_{A} \geq 0
\end{gathered}
$$

The moment $\boldsymbol{M}_{\boldsymbol{A}}=\boldsymbol{A B} \times \boldsymbol{R}_{34}$ is calculated using the vectors $\boldsymbol{A B}$ and $\boldsymbol{R}_{34}$.

$\boldsymbol{A} \boldsymbol{B}$ is determined from the position analysis of the mechanism (Appendix)

$\boldsymbol{R}_{34}$ can be obtained when solving the matrix Equation in the Appendix.

\subsection{Optimization results}

The optimum parameters are found to be: $b_{3}=0.385 \mathrm{~m}$, $b_{4}=0.030 \mathrm{~m}$. The required input torques for this optimum case along with the case when there is no spring attached, are shown in Fig. 16. Compared to the value of the torque with no spring, the torque corresponding to the optimized solution maintained similar values. 


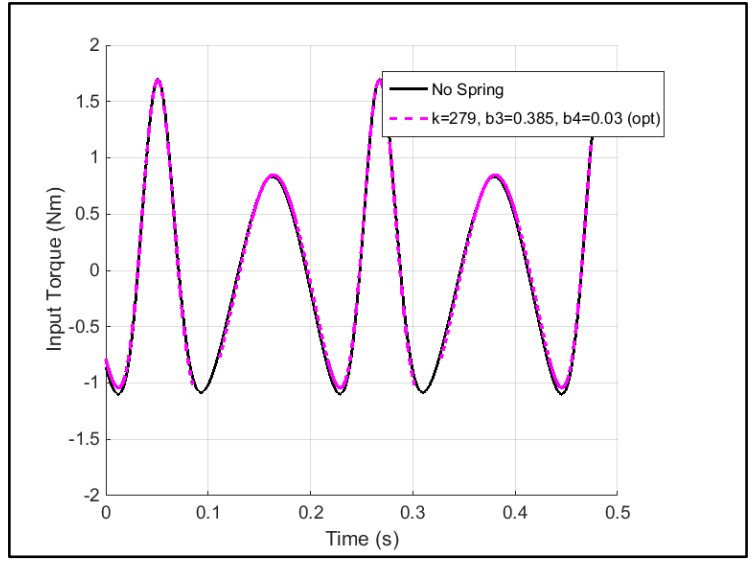

Fig. 16: Input torque with and without a spring

The variation of the moment shown on Fig. 17 indicates a change of the direction of this moment. This change causes impacts during the motion. When the spring is attached at the optimum location given above, the moment keeps the same direction during the motion, which will insure that there will be no impact.

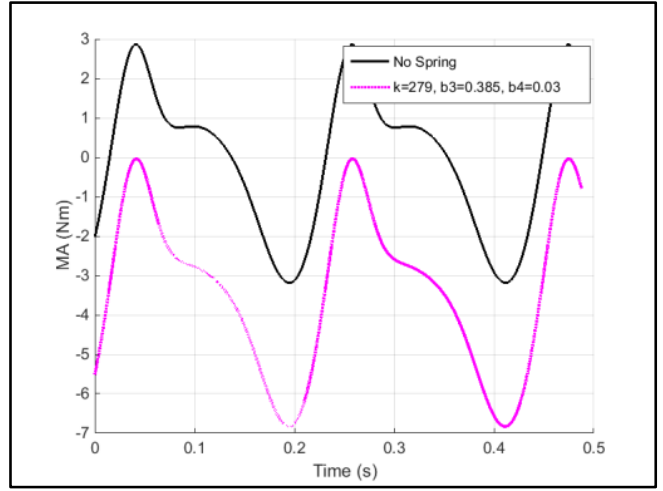

Fig. 17: Moment of the reaction force before and after optimization

Experimental results confirm these findings. Fig. 18 shows the acceleration of point $\mathrm{D}$ (the location of the accelerometer), for a duration of $0.5 \mathrm{~s}$, in three cases: the first case corresponds to the mechanism without a spring. The maximum acceleration reaches a value of $400 \mathrm{~m} / \mathrm{s}^{2}$. The second case represents the acceleration of point $\mathrm{D}$ when a spring is attached in the middle of the coupler and the follower. The peaks in this case increased to around 600 $\mathrm{m} / \mathrm{s}^{2}$. The third case corresponds to the spring in its optimum location. The maximum value of the acceleration drops to less than $200 \mathrm{~m} / \mathrm{s}^{2}$, in this case.

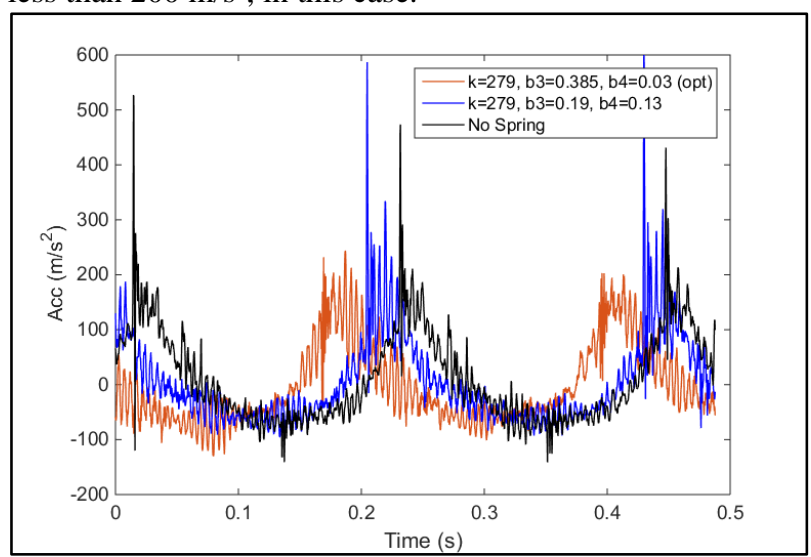

Fig. 18: Experimental results at $29 \mathrm{rad} / \mathrm{s}(277 \mathrm{rpm})$

Fig. 19 shows the simulation of the mechanism along with the vector representing the reaction force in the joint with clearance, when the spring is attached in its optimum location. It is worth mentioning that the direction of the reaction force is mostly pointing toward the center of the pin joint of link 4 with the ground. The important remark is that the reaction force is kept on only one side of link 3 , which generated a negative moment around point $\mathrm{A}$, at all times. The experimental results (Fig. 18) confirm this conclusion.

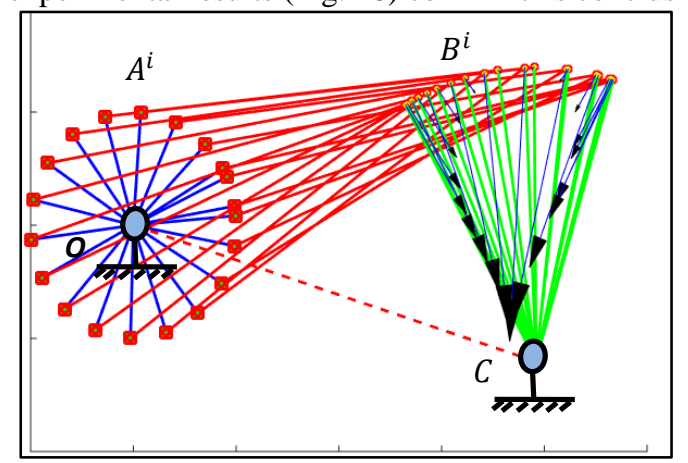

Fig. 19: The magnitude and the orientation of the reaction force during the motion

Fig. 20 shows the trajectory of the center of the joint with clearance obtained using ADAMS model with a spring. It is clear that the contact between the journal and the sleeve is always maintained, which eliminated any impact. The penetration is due mainly to the high reaction forces, which could be a source of excessive wear in the joint. Indeed, compared to the case without a spring the average of the magnitudes of the reaction force in the case with a spring (Fig. 19) is higher, which could explain the penetration shown in Fig. 20.
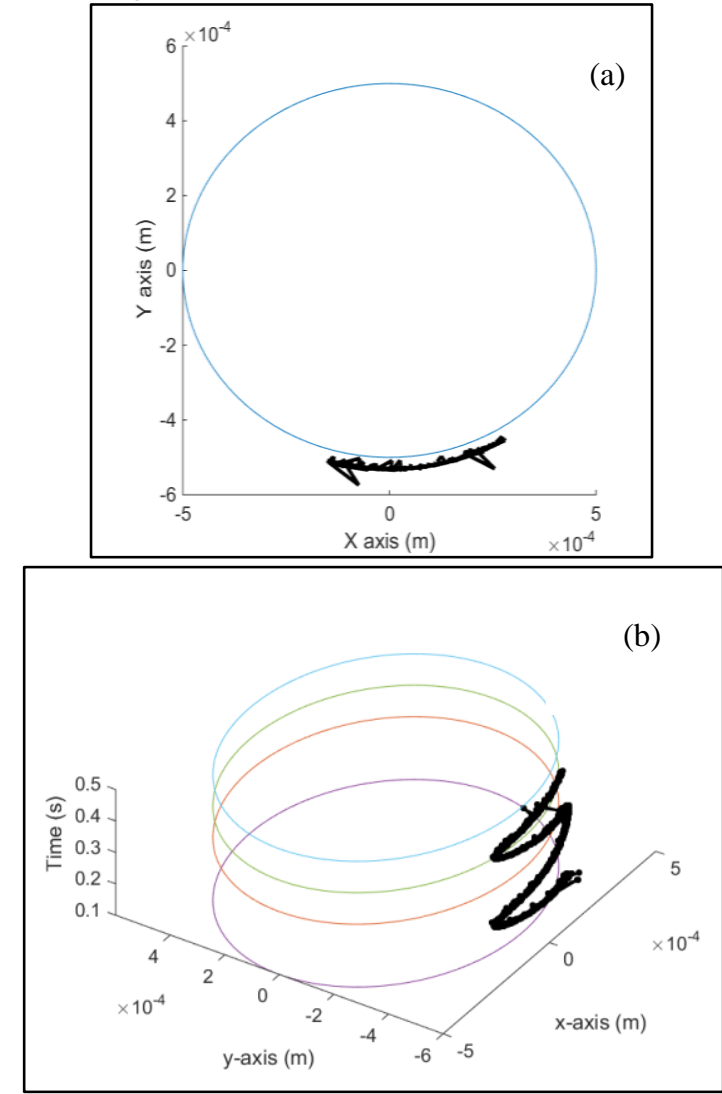

Fig. 20: ADAMS model with a spring: journal center trajectory (a) XY plot, (b) XY-time plot (the circles represent the clearance) 


\subsection{Effect of the speed}

The optimum location of the spring was calculated for a speed of $277 \mathrm{rpm}\left(\mathrm{N}_{0}\right)$. As it was shown in Fig. 18, the spring succeeded in eliminating the impacts in the joint with clearance. Two more speeds $\left(\mathrm{N}_{1}=415 \mathrm{rpm}\right.$ and $\left.\mathrm{N}_{2}=554 \mathrm{rpm}\right)$ were checked experimentally to determine if the spring is still capable of eliminating the impacts (Fig. 21, Fig. 23). ADAMS simulations are also run for these two speeds (Fig. 22, Fig. 24). It is worth mentioning that for the first case (415 $\mathrm{rpm})$ the spring reduced drastically the peaks due to the impacts. Some small peaks still persist, however, which are clear in Fig. 23. For the case of high speed (554 rpm) the spring has less effect on the peaks, but it still reduced the effect of the impacts.
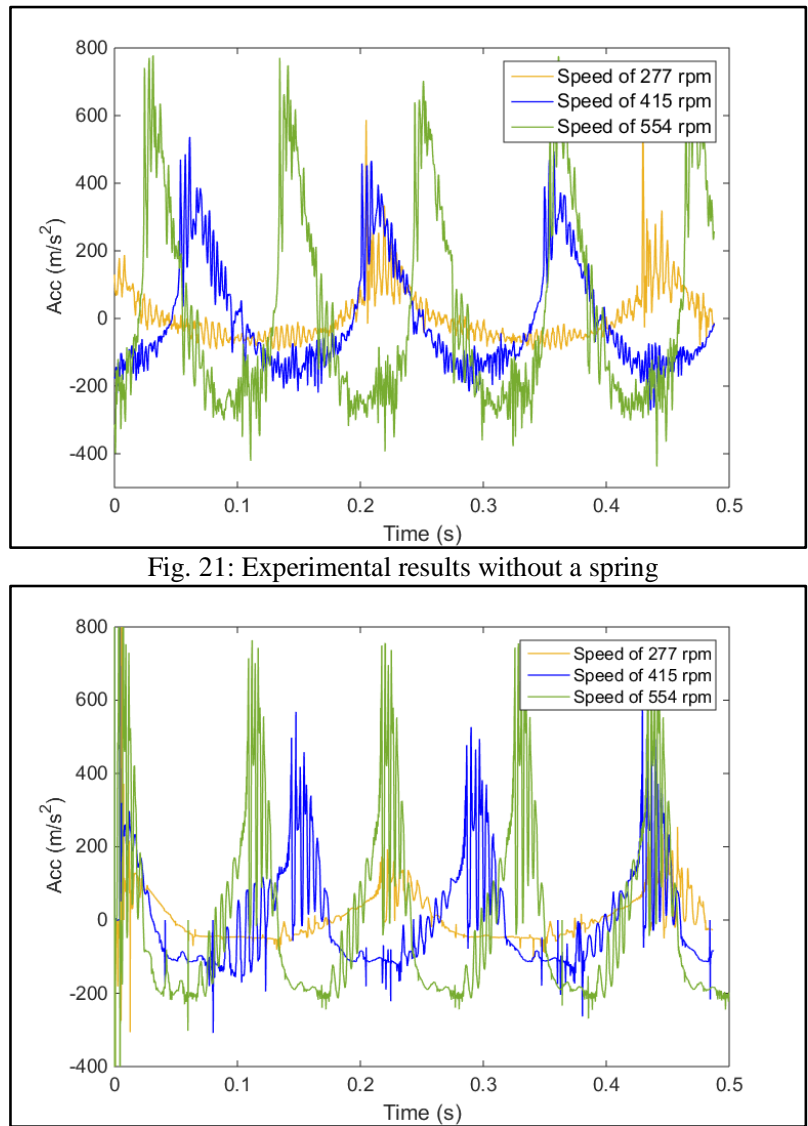

Fig. 22: ADAMS results without a spring

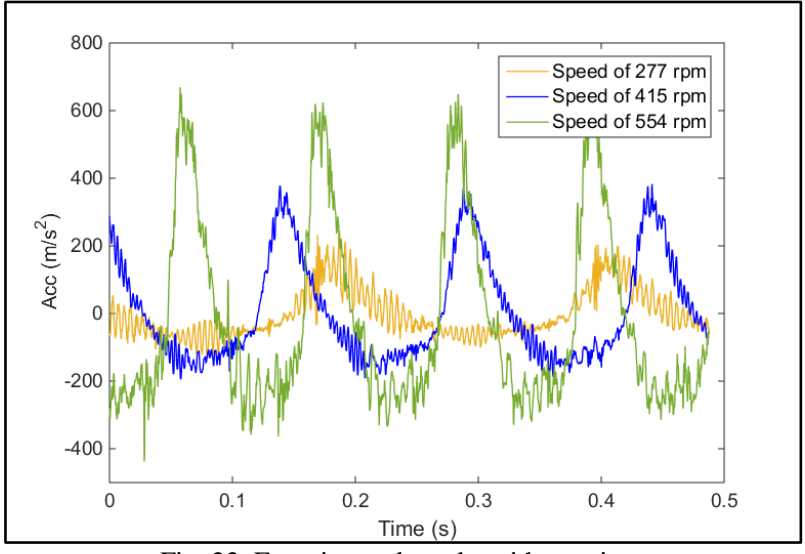

Fig. 23: Experimental results with a spring

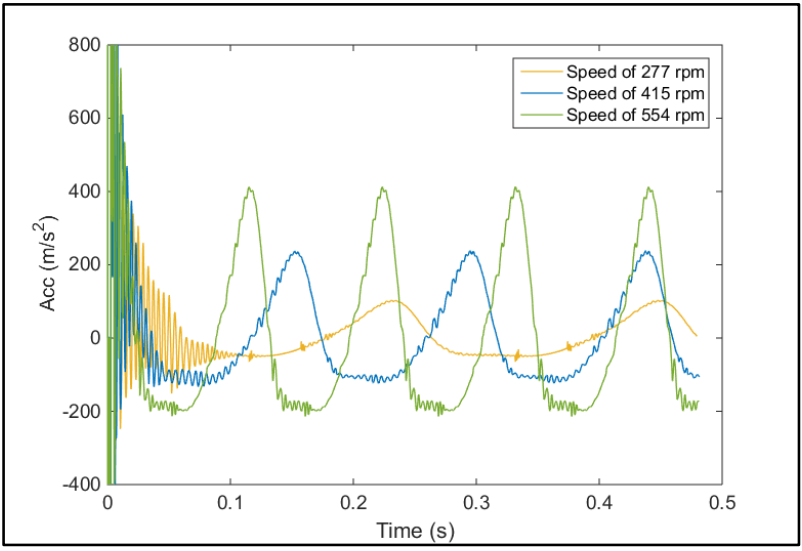

Fig. 24: ADAMS results with a spring

\section{Conclusion}

In this work, we investigated the dynamic behavior of a planar four-bar mechanism. By monitoring the moment of the reaction force in the joint with clearance, a simple yet effective condition was derived to predict the occurrence of the impact in the joint. Two impacts were identified per rotation of the crank. An experimental setup was built to validate this result. A model under ADAMS confirmed the existence of three types of motion, i.e., continuous contact, free flight and impact. To eliminate these impacts, a simple solution is proposed, which is based on adding a spring to the system. It was shown that the impact could be easily eliminated using adequate spring parameters. However, this modification resulted in a higher maximum input torque and an increase of its fluctuation. To keep the beneficial effect of the spring and minimize its bad effect on the torque, an optimization, using MATLAB, was performed. The objective function to be minimized was the maximum value of the torque under the constraint of keeping the moment of the reaction force always in one direction. The obtained results were simulated and they showed a net improvement of the dynamic behavior of the system, compared to the nonoptimized case. The model under ADAMS and the experimental results confirmed the results of the simulations. The system was checked experimentally for two more speeds and it was shown that the spring had a partial effect in the case of high speed.

\section{Acknowledgement}

This work was financed by the American University of Sharjah under the grant number: FRG15-R-40

\section{Bibliography}

[1] P. Flores, H.M. and Lankarani, Dynamic Response of Multibody Systems with Multiple Clearance Joints, Transactions of The ASME: Journal of Computational and Nonlinear Dynamics, (7) (2012) 1-13.

[2] S.M. Megahed and A.F. Haroun, Analysis Of The Dynamic Behavioral Performance Of Mechanical Systems With Multi-Clearance Joints, Transactions Of The ASME: Journal Of Computational And Nonlinear Dynamics, (7) (2012)

[3] I. Khemili and L. Romdhane, Dynamic Analysis of A Flexible Slider-Crank Mechanism with Clearance, European Journal Of Mechanics A/Solids (27) (2008) 882-898

[4] S. Erkaya, S. Doğan and Ş. Ulus, Effects Of Joint Clearance On The Dynamics Of A Partly Compliant 
Mechanism: Numerical And Experimental Studies, Mechanism And Machine Theory (88) (2015) 125-140

[5] X. Yao, X. Guo, Y. Feng, C. Yu and C. Ma, Dynamic Analysis for Planar Beam with Clearance Joint, Journal Of Sound And Vibration, (339) (2015) 324-341

[6] Y. M. Dardel and H.M. Daniali, Nonlinear Dynamics And Control Of Crank-Slider Mechanism With Link Flexibility And Joint Clearance, Proceedings Of The Institution Of Mechanical Engineers, Part C: Journal Of Mechanical Engineering Science, (230) (2015) 737 755.

[7] M. Machado, J. Cost, E. Seabr and Paulo Flores, The Effect Of The Lubricated Revolute Joint Parameters And Hydrodynamic Force Models On The Dynamic Response Of Planar Multibody Systems, Nonlinear Dynamics, (69) (2012) 635-654

[8] A. Azimi Olyaei and M. R. Ghazavi, Stabilizing SliderCrank Mechanism with Clearance Joints, Mech. Mach. Theory, (53) (2012) 17-29.

[9] J. L. Ha, R. F. Fung, K. Y. Chen and S. C. Hsien, Dynamic Modeling and Identification Of A SliderCrank Mechanism, J. Sound Vib., (289) (2006) 10191044.

[10] E. Zheng and X. Zhou, Modeling and Simulation Of Flexible Slider-Crank Mechanism With Clearance For A Closed High Speed Press System, Mech. Mach. Theory, (74) (2014) 10-30.

[11] S. Erkaya and İ. Uzmay, Experimental Investigation of Joint Clearance Effects On the Dynamics of A SliderCrank Mechanism, Multibody System Dynamics, (24) (2010) 81-102

[12] İ. Uzmay and S. Erkaya, Investigation On Effect of Joint Clearance On Dynamics of Four-Bar Mechanism, Nonlinear Dynamics, (58) (2009) 179-198

[13] P. Flores, C.S. Koshy, N.M. Lankarani, J, Ambrosio, and J.C.P. Claro, Numerical and Experimental Investigation On Multibody Systems With Revolute Clearance Joints. Nonlin. Dyn. (65) (2011) 383-398

[14] F. Meng, S. Wu, F. Zhang and L. Liang, Numerical Modeling and Experimental Verification For HighSpeed And Heavy-Load Planar Mechanism With Multiple Clearances, Mathematical Problems In Engineering, (2015),

[15] P. Flores and J. Ambrósio, Revolute Joints with Clearance in Multibody Systems. Comput. Struct. (82) (2004) 1359-1369

[16] P. Flores and J. Ambrósio, On the Contact Detection for Contact-Impact Analysis in Multibody Systems, Multibody Syst. Dyn., (24) (2010) 103-122.

[17] Z. Hai-Yang, X. Min-Qiang, W. Jin-Dong, and L. Yong-Bo, A Parameters Optimization Method for Planar Joint Clearance Model and Its Application for Dynamics Simulation of Reciprocating Compressor, $J$. Sound Vib., (344) (2015) 416-433.

[18] S. Erkaya and S. Doğan, A Comparative Analysis of Joint Clearance Effects on Articulated and Partly Compliant Mechanisms, Nonlinear Dynamics, (81) (2015) 323-341

[19] Z. Zhang, L. Xu, Y.Y. Tay, P. Flores And H. Lankarani, Multi-Objective Optimization Of Mechanisms With Clearances In Revolute Joints, In New Trends In Mechanism And Machine Science, Mechanisms And Machine Science 24, P. Flores And F. Viadero (Eds.), Springer International Publishing Switzerland 2015
[20] S.M. Varedi, H.M. Daniali, M. Dardel, A. Fathi, Optimal Dynamic Design of A Planar Slider-Crank Mechanism With A Joint Clearance, Mechanism And Machine Theory (86) (2015) 191-200

[21] S. Erkaya and İ. Uzmay, Optimization Of Transmission Angle For Slider-Crank Mechanism With Joint Clearances, Structural And Multidisciplinary Optimization, (37) (2009) 493-508

[22] S. Erkaya and İ. Uzmay, "A Neural-Genetic (NN-Ga) Approach for Optimising Mechanisms Having Joints with Clearance", Multibody System Dynamics, (20) (2008) 69-83

[23] S. Erkaya, "Trajectory Optimization Of A Walking Mechanism Having Revolute Joints With Clearance Using ANFIS Approach", Nonlinear Dynamics, (71) (2013) 75-91,

[24] B. Feng, N. Morita, T. Torii: A New Optimization Method for Dynamic Design of Planar Linkage with Clearances at Joints-Optimizing the Mass Distribution of Links to Reduce the Change of Joint Forces. J. Mech. Des., Trans. ASME. (124) (2002) 68-73

[25] S. Erkaya and İ. Uzmay, "Determining Link Parameters Using Genetic Algorithm In Mechanisms With Joint Clearance", Mechanism And Machine Theory, (44) (2009) 222-234,

[26] G. Huixin, Y. Wenhui, Design Optimization of Planar Linkage Mechanism with Joint Clearance for Improving the Robustness of Kinematic Accuracy, Journal of Mechanical Engineering, (03) (2012)

\section{Appendix}

The kinematic model of the 4-bar mechanism of Fig. 1 is given by:

\section{Position:}

In a complex form:

$L_{2} e^{\mathrm{i} \theta_{2}}+L_{3} e^{\mathrm{i} \theta_{3}}=L_{1} e^{\mathrm{i} \theta_{1}}+L_{4} e^{\mathrm{i} \theta_{4}}$

In a scalar form:

$L_{2} \cos \theta_{2}+L_{3} \cos \theta_{3}=L_{1} \cos \theta_{1}+L_{4} \cos \theta_{4} \quad$ (A1a)

$L_{2} \sin \theta_{2}+L_{3} \sin \theta_{3}=L_{1} \sin \theta_{1}+L_{4} \sin \theta_{4} \quad$ (A1b)

The input is $\theta_{2}$ and the unknowns are $\theta_{3}$ and $\theta_{4}$. Since $\theta_{1}$ is constant.

$\mathbf{A B}=L_{3} e^{\mathrm{i} \theta_{3}}=L_{3} \cos \theta_{3} \mathbf{x}+L_{3} \sin \theta_{3} \mathbf{y}$

Velocity:

$-L_{2} \dot{\theta}_{2} \sin \theta_{2}-L_{3} \dot{\theta}_{3} \sin \theta_{3}=-L_{4} \dot{\theta}_{4} \sin \theta_{4} \quad$ (A2a)

$L_{2} \dot{\theta}_{2} \cos \theta_{2}+L_{3} \dot{\theta}_{3} \cos \theta_{3}=L_{4} \dot{\theta}_{4} \cos \theta_{4} \quad$ (A2b)

The input is a constant $\dot{\Theta}_{2}$ and the unknowns are $\dot{\Theta}_{3}$ and $\dot{\Theta}_{4}$. Acceleration:

$-L_{2} \dot{\theta}_{2}{ }^{2} \cos \theta_{2}-L_{3} \ddot{\theta}_{3} \sin \theta_{3}-L_{3} \dot{\theta}_{3}{ }^{2} \cos \theta_{3}=$

$-L_{4} \ddot{\theta}_{4} \sin \theta_{4}-L_{4} \dot{\theta}_{4}^{2} \cos \theta_{4}$

$-L_{2} \dot{\theta}_{2}{ }^{2} \sin \theta_{2}+L_{3} \ddot{\theta}_{3} \cos \theta_{3}-L_{3} \dot{\theta}_{3}{ }^{2} \sin \theta_{3}=$

$L_{4} \ddot{\theta}_{4} \cos \theta_{4}-L_{4} \dot{\theta}_{4}^{2} \sin \theta_{4}$

The unknowns are $\ddot{\theta}_{3}$ and $\ddot{\Theta}_{4}$.

\section{Kinetostatic model}

Newton's law applied to the three moving links, yields 9 equations with 9 unknowns, which are the 8 reaction forces and the input torque. The following set of linear equations is solved to obtain these unknowns: 


$\left[\begin{array}{ccccccccc}1 & 0 & 1 & 0 & 0 & 0 & 0 & 0 & 0 \\ 0 & 1 & 0 & 1 & 0 & 0 & 0 & 0 & 0 \\ 0 & 0 & L_{2} s_{2} & -L_{2} c_{2} & 0 & 0 & 0 & 0 & 1 \\ 0 & 0 & -1 & 0 & 1 & 0 & 0 & 0 & 0 \\ 0 & 0 & 0 & -1 & 0 & 1 & 0 & 0 & 0 \\ 0 & 0 & -r_{3} c_{3} & r_{3} s_{3} & \left(L_{3}-r_{3}\right) s_{3} & \left(L_{3}-r_{3}\right) c_{3} & 0 & 0 & 0 \\ 0 & 0 & 0 & 0 & -1 & 0 & 1 & 0 & 0 \\ 0 & 0 & 0 & 0 & 0 & -1 & 0 & 1 & 0 \\ 0 & 0 & 0 & 0 & L_{4} s_{4} & -L_{4} c_{4} & 0 & 0 & 0\end{array}\right]\left[\begin{array}{c}R_{12}^{x} \\ R_{12}^{y} \\ R_{32}^{x} \\ R_{32}^{y} \\ R_{34}^{x} \\ R_{34}^{y} \\ R_{14}^{x} \\ R_{14}^{y} \\ T_{2}\end{array}\right]=\left[\begin{array}{c}m_{2} a_{G 2}^{x} \\ m_{2} a_{G 2}^{y} \\ 0 \\ m_{3} a_{G 3}^{x}-F_{s}^{x} \\ m_{3} a_{G 3}^{y}-F_{s}^{y} \\ I_{3} \ddot{\theta}_{3}+T_{s 3} \\ m_{4} a_{G 4}^{x}+F_{s}^{x} \\ m_{4} a_{G 4}^{y}+F_{s}^{y} \\ I_{C 4} \ddot{\theta}_{4}+T_{s 4}\end{array}\right]$

$s_{i} \equiv \sin \left(\theta_{i}\right)$ and $c_{i} \equiv \cos \left(\theta_{i}\right)$

$a_{G i}$ is the acceleration of the center of mass of link $i$.

The spring force is given as follows:

$\mathbf{F}_{s}=k\left(S-S_{0}\right) e^{i \theta_{s}}, T_{s 3} \mathbf{z}=\mathbf{F}_{s} \times \mathbf{G}_{3} \mathbf{B}_{3}$ and $T_{s 4} \mathbf{z}=\mathbf{F}_{s} \times \mathbf{C B}_{4}$

where $S=B_{3} B_{4}$ and $\theta_{S}=\operatorname{atan} 2\left(y_{B_{3} B_{4}}, x_{B_{3} B_{4}}\right)$

Each three rows of the matrix represent the three equilibrium equations of one of the links. 